\title{
Automatic Thresholding TeCHNiQues FOR SAR IMAGES
}

\author{
Moumena Al-Bayati and Ali El-Zaart \\ Department of Mathematics and Computer Science, Beirut Arab University \\ Beirut, Lebanon \\ Moumena.alhadithi@yahoo.com \\ elzaart@bau.edu. lb
}

\begin{abstract}
Segmentation of Synthetic Aperture Radar (SAR) images have a great use in observing the global environment, and in analysing the target detection and recognition. But, segmentation of (SAR) images is known as a very complex task, due to the existence of speckle noise. Therefore, in this paper we present a fast SAR images segmentation based on between class variance. Our choice for used $(B C V)$ method, because it is one of the most effective thresholding techniques for most real world images with regard to uniformity and shape measures. Our experiments will be as a test to determine which technique is effective in thresholding (extraction) the oil spill for numerous SAR images, and in the future these thresholding techniques can be very useful in detection objects in other SAR images.
\end{abstract}

\section{KEYWORDS}

SAR IMAGES, SEGMENTATION, THRESHOLDING, OTSU METHOD .

\section{INTRODUCTION}

Segmentation is a critical preprocess step in image processing and pattern recognition $[9,13]$. Segmentation is considered as very essential role in analysis many applications such as Synthetic Aperture Radar (SAR) image. Commonly, segmentation of SAR images classify into two classes; one based on gray levels and the other based on texture [2,3]. The main obstacle that faced the segmentation of (SAR) images is the speckle noise [2,3]. Speckle noise is a phenomenon appeared in all imaging system. The reason of forming this noise is attributed to random conflict between coherent returns came from many scatters appear on a surface, on the scale of a wavelength of the incident radar wave[2]. Speckle noise has two negative effects (1) it decreases the ability to determine fine details, (2) it makes the segmentation work very difficult. Therefore, many approaches have been presented and applied to improve the segmentation of SAR images [1,2]. Like [4]Yongmin Shuai, Hong Sun, Ge Xu introduced SAR Image Segmentation Based on Level Set. In [5] Xiaoli Tian, Shuiping Gou and Licheng Jiao improve SAR image segmentation technique based on spatially adaptive weighted Possibilistic c- means clustering . In [2]Ghada Al-Osaimi and Ali El-Zaart propose Minimum Cross Entropy Thresholding for SAR Images. [6] Haiyan Li and Zhengyao Bai present a new PCNN-Based method for segmentation of SAR images. [7] H. B. Kekre, Saylee Gharge, and Tanuja K. segment SAR Image using vector quantization technique on entropy images. [15] Xian-Bin Wen, Hua Zhang and Fa-Yu Wang apply A Wavelet Neural Network for SAR Image Segmentation. [16] Lei Hu, Yang Li and Feng Gao implement Kullback -leibler distance of edge worth to segment (SAR).[17] Shiqi Huang introduces an improved unsupervised threshold determination method for SAR image change detection. Our contribution in this work is to introduce five automatic 
thresholding techniques for SAR images. The selection of their optimal threshold will be based on maximization the separability factor between the classes.

Thresholding is a simple and most spread technique of segmentation. It based on classification image pixels into object and background depended on the relation between the gray level value of the pixels and the threshold $[8,9,10,11,12]$.

In general, there are two types of thresholding. (1) Global thresholding used for images have good illumination. It has one value to segment an entire image. Global threshold is simple, easy to apply, and faster due to its less need for computations. (2) local thresholding is used for images with non-uniform illumination. By using the local thresholding; an entire image will be segmented into sub segments; in each small segment there is a specific threshold. Local thresholding is identified with its complexity, difficulty, and it is slow in the implementation[9]. On the other hand, Sankur [14] presents another classification of thresholding, but he classified thresholding techniques depended on the information they are exploiting : such as histogram shape, entropy, object attributes, spatial correlation, local gray-level surface, and clustering of gray-level information.

This paper presents Otsu method and all the techniques that related to it. Otsu method classified within clustering of gray-level information. Moreover, it is one of the most popular and effective image thresholding technique, so that many thresholding techniques have been constructed to revise Otsu method; each technique improved Otsu method in a specific way like [9] valley emphasis technique, [10] neighborhood valley emphasis technique, [11] variance and intensity contrast, and [12] variance discrepancy technique.

The rest of the paper is organized as follows: Section 2 is about the formulas used in thresholding. Section3 describes Otsu method and all the methods related to it. Thresholding evaluation methods are presented in Section 4. Experimental results for (SAR) images are in Section 5. Section 6 concludes the work.

\section{FORMULATION}

Assume the pixels of the image be described by $L$ gray levels $[0, \ldots, L-1]$. The number of pixels in level $\mathrm{i}$ is represented by $n_{i}$, and $\mathrm{n}$ is the total number of pixels. $[8,9,10,11,12]$.

$$
\mathrm{n}=\sum^{1-2} \mathrm{n}_{1}
$$

Gray level histogram is normalized and regarded as a probability distribution:

$$
h_{i}=\frac{n_{1}}{n}
$$

Suppose we divide the pixels into two classes $C_{1}$ and $C_{2}$ by the threshold t. The first class $C_{1}$ represent pixels with levels $[0, . ., \mathrm{t}]$, and $C_{2}$ represent pixels $[\mathrm{t}+1, . . \mathrm{L}-1]$.

The probability of occurrence of the two classes can be represented as the following:

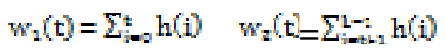

The mean and variance of the background and object are represented respectively as the following : 


$$
\begin{aligned}
& \mu_{2}(\mathrm{t})=\Sigma_{t=0}^{ \pm} \mathrm{i} \mathrm{h}(\mathrm{i}), \sigma_{2}^{z}(\mathrm{t})=\Sigma_{i=0}^{t}\left(\mathrm{i}-\mu_{2}(\mathrm{t})\right)^{\mathrm{z}} \mathrm{h}(\mathrm{i}) \\
& \mu_{z}(\mathrm{t})=\Sigma^{1-1} \mathrm{i} h(\mathrm{i}), \sigma_{z}^{2}(\mathrm{t})=\Sigma_{\mathrm{ten+1}}^{1-1}\left(\mathrm{i}-\mu_{2}(\mathrm{t})\right)^{\mathrm{z}} \mathrm{h}(\mathrm{i})
\end{aligned}
$$

\section{OTSU METHOD}

Otsu assumes maximizing the weight sum of between class variances is equivalent to minimizing within class variance[8]. The next equations will denote to within-class variance and between class variance respectively:

$\sigma_{w}^{2}(t)=\omega_{2}(t) \sigma_{2}^{2}(t)+\omega_{2}(t) v_{2}^{2}(t)$

$\sigma_{E}^{2}(t)=\omega_{2}(t)\left(\mu_{2}(t)-\mu_{T}(t)\right)^{2}+\omega_{2}\left(\mu_{2}(t)-\mu_{T}(t)\right)^{2}$

The final form of between-class variance can be formulated as the following :

$\left.\sigma_{z}^{2}(t)=\omega_{1}(t) \omega_{z}(t)\left(\mu_{z}: t\right)-\mu_{2}(t)\right)^{2}$

The algorithm of Otsu method is :

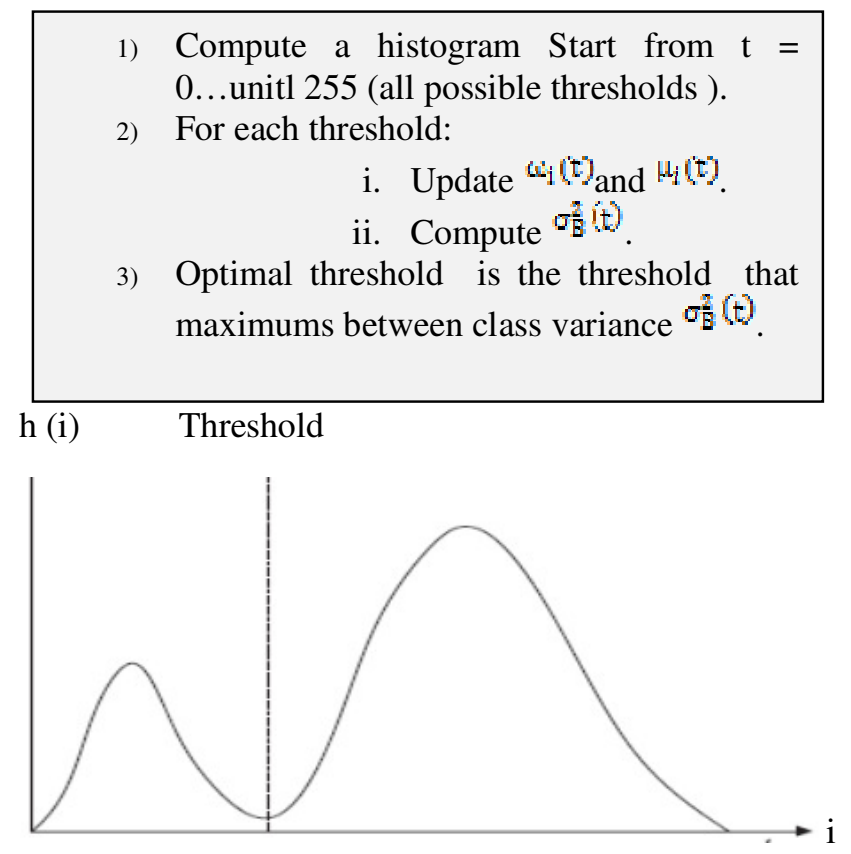

Figure 1. Optimal threshold in the histogram [9]

\subsection{Valley Emphasis Method}

Valley emphasis method is used to determine optimal threshold values for both bimodal and unimodal distribution. It succeeds in detection large and small objects. This method implements a new weight to confirm that the optimal threshold is in the valley between two packs for (bimodal histogram), or at the bottom rim for (unimodal histogram).

In this method the optimal threshold has two factors: (1) it has smaller probability of occurrence. (2) it maximizes between class variance as in Otsu method [9].

The valley-emphasis equation is as in [9]. 


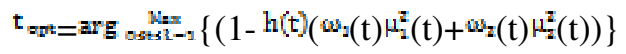

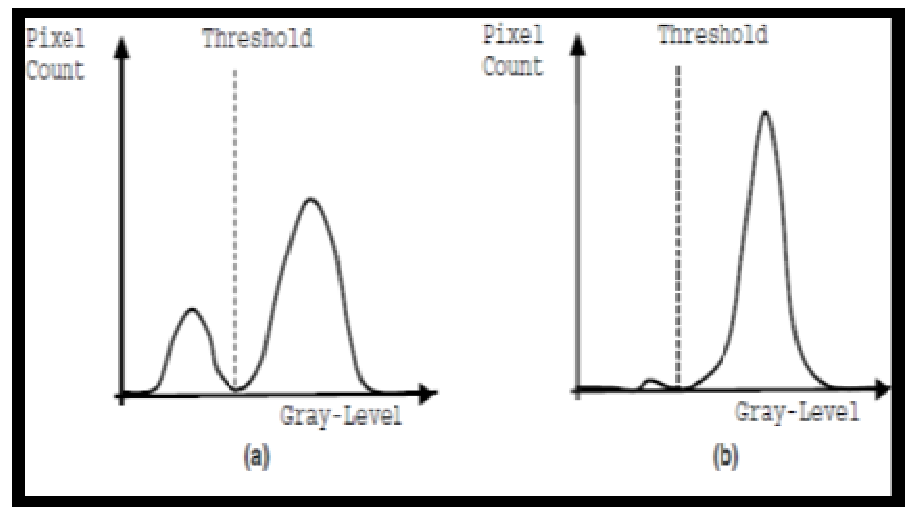

Figure 2 Optimal thresholding selection in gray-level histogram: (a) bimodal; (b) unimodal [10]

\subsection{Neighborhood Valley Emphasis Method}

Neighbourhood valley emphasis method is a modified for the prior method (valley emphasis method). In this method between class variance $\sigma_{\mathrm{B}}^{2}(\mathrm{t})$ is computed for both the valley point and its neighborhood. It used for thresholded images that have large diversity between the object variance and background variance.

The sum of neighborhood gray level value $\mathrm{h}(\mathrm{i})$ denotes within the interval $\mathrm{n}=2 \mathrm{~m}+1$ for gray level $\mathrm{i}, \mathrm{n}$ denotes to the number of neighborhood that should be odd number.

If the image has one dimensional histogram $\mathrm{h}(\mathrm{i})$; the neighborhood gray value $\overline{\mathrm{h}}(\mathrm{i})$ of the grey level $\mathrm{i}$ is denoted as:

$\bar{h}(i)=[h(i-m)+\ldots+h(i-1)+h(i)+h(i+1)+\ldots+h(i+m)]$

Neighborhood valley emphasis method is represented as the following :

$\xi(\mathrm{t})=(1-\overline{\mathrm{h}}(\mathrm{t}))\left(\left(\omega_{\mathrm{z}}(\mathrm{t}) \mu_{1}^{\mathrm{z}}(\mathrm{t})+\omega_{\mathrm{z}}(\mathrm{t}) \mu_{\mathrm{z}}^{\mathrm{z}}(\mathrm{t})\right)\right.$

In the next Eq. (12) the first part denotes to the maximum weight of the valley point and its neighborhood, while the second part denotes the maximum between class variance[10].

The optimal threshold is :

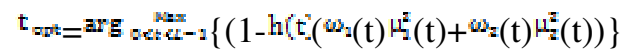




\subsection{Thresholding Based on Variance and Intensity Contrast}

The new formula is presented for detection small objects from complex homogeneity background. It computes the weighted sum of within class variance and the intensity contrast between the object and background simultaneously.

The new formula is as the following :

$\mathrm{J}(\lambda, \mathrm{t})=(1-\lambda) \sigma_{\mathrm{w}}(\mathrm{t})-\lambda\left|\mu_{2}(\mathrm{t})-\mu_{\mathrm{z}}(\mathrm{t})\right|$

$(t), \beta_{2}(t)$ are the mean intensities of the object and background. $\sigma_{W}(t)$ is the square root of withinclass variance. $\sigma_{N}(t)$ is formulated from the following equation.

$$
a_{1}^{2}\left(t^{2}=w_{1}(t) a_{1}^{2}\left(t^{2}+w_{1}(t) \sigma_{2}^{2}(t)\right.\right.
$$

Where, the first part represents the probability of class occurrence and the standard deviation (variance) of the first class, while the second part represents the probability of class occurrence and the standard deviation (variance) of the second class.

In the presented formula within class variance determines the intensity homogeneity within the object and background, but the intensity contrast captures the intensity contrast between them. $A$ factor is a weight that determines and balances the contribution of (within class variance , intensity contrast) in the formula. ${ }^{\lambda}$ Values are within the range $[0,1)$. When ${ }^{\dot{\alpha}}=0$ the optimal threshold will be determined from the within class variance, but if $\lambda=1$ the new formula tends to the intensity contrast [11].

\subsection{Variance Discrepancy Method}

A presented method used to segment images have large variance discrepancy between the object and background. It takes into account both class variance sum and variances discrepancy at the same time. It is formulated as the following :

$I(\alpha, t)=\alpha\left(\sigma_{2}^{2}(t)+\sigma_{2}^{z}\left(t^{2}\right)+(1-\alpha) \sigma_{D}\left(t^{2}\right)\right.$

Where

$\sigma_{p}(t)=\sigma_{2}(t) \sigma_{2}(t)$

and. $\sigma_{1}^{2}(\mathrm{t})<=\sigma_{Z}(\mathrm{t})<=\sigma_{2}^{2}(\mathrm{t})$ or $\sigma_{2}^{2}(\mathrm{t})<=\sigma_{Z}(\mathrm{t})<=\sigma_{1}^{2}(\mathrm{t}), \sigma_{D}(\mathrm{t})$ Is a measurement of variance discrepancy of (object, background), while $\sigma_{1}^{2}(t), \sigma_{2}^{2}(t)$ are represent the standard deviation (variances) of the two classes.

In the presented method the factor $\alpha$ is an important factor. It balances the contributions of class variance sum and variance discrepancy in the method. $\alpha$ values is within the range $[0,1]$. When $\alpha$ is large the method degenerates to class variance sum, which make class variance discrepancy has limited effect. On the contrary, if $\alpha$ is small the method will be based on class variance discrepancy, and the effect of class variance sum will be neglected [12].

\section{THRESHOLDING EVALUATION METHODS}

The quality of thresholding technique is a critical issue; it various depending on the type of the thresholding technique and the kind of image. In order to analyse the performance of the thresholding techniques, there are different evaluation methods used to measure the efficiency of 
the thresholding techniques. These evaluation methods will normalize the results within the range $[0, \ldots, 1]$. Zero denote to well thresholded image, and one denote to totally incorrect thresholded image. In our study we used two evaluation methods Region Non-Uniformity (NU) and InterRegion Contrast (GC) [18]. These methods help us to determine which technique is the best in extraction the region of interest (object) from the background.

\subsection{Region Non-Uniformity (NU)}

This method measures the ability to distinguish between the background and object in the thresholded image. In the following NU equation $\sigma^{2}(t)$ refers to the variance of the whole image, while $\sigma_{0}^{2}(t)$ refers to the variance of the object (foreground). $w_{0}(t)$ represents the probability of occurrence of the object. Region non-uniformity equal to zero denotes to perfect thresholded image, but NU $=1$ denotes for mixed areas between the object and background [18].

$$
\mathrm{NU}=\frac{w_{0}(\mathrm{t}) \sigma_{0}^{2}(\mathrm{t})}{\sigma^{2}(\mathrm{t})}
$$

\subsection{Inter -Region Contrast (GC)}

This method is very important in measure the contrast degree in the thresholded image. A good thresholded image should have higher contrast across adjacent regions. In the following GC equation the object average gray-level is known as $\mu_{0}(t)$, and the background average gray-level is known as $\mu_{b}(t)[18]$.

$$
\mathrm{GC}=1-\frac{\mu_{0}(\mathrm{t})-\mu_{\mathrm{b}}(\mathrm{t})}{\mu_{\mathrm{g}}(\mathrm{t})+\mu_{\mathrm{b}}(\mathrm{t})}
$$

\section{EXPERIMENTAL RESULTS}

In this section, we implement five different thresholding techniques on numerous SAR images using Gaussian distribution. Fig.3(a) and Fig.4(a) are high contrast SAR images. In general, we found that the five thresholding techniques in Fig.3 (b, c, d, e, f) give good results, but the smallest value of region non uniformity is get from Otsu method NU $=0.229594$, and the smallest inter region contrast is obtained from neighbourhood valley emphasis method GC $=0.445856$. In Fig.4 (b, c, d, e, f) also present satisfactory results, and all of them detect the small details of the objects (oil) from the background. Although, the smallest region non uniformity is presented from Otsu method NU $=0.202736$, while the best inter region contrast is obtained from neighbourhood valley emphasis method GC $=0.473041$. Similarly, we did with two low contrast SAR images in Fig.5 and Fig.6. We compare the results of the five thresholding techniques, and we found that for Fig.5 (b, c, d, e, f) the smallest region non uniformity is get from Otsu method NU=0.191687, and the smallest inter region contrast is obtained from neighbourhood valley emphasis method $\mathrm{GC}=0.502545$. Finally, Fig.6 (b, c, d, e, d) the five thresholding techniques separate the object from the background successfully. However, we select the minimum value of region non uniformity from Otsu method $\mathrm{NU}=0.192322$, and the minimum inter region contrast is selected from neighbourhood valley emphasis method $\mathrm{GC}=0.563509$.

It is notable that the results of variance and intensity contrast method are similar to the good results for Otsu method when we choose $\lambda=0$ or 0.05 . 
Table I. shows ( T, NU, GC, AV) values of five thresholding techniques

\begin{tabular}{|c|c|c|c|c|c|}
\hline & & Fig. 3 & Fig. 4 & Fig.5 & Fig.6 \\
\hline \multirow{4}{*}{ Otsu } & $\mathrm{T}$ & 42 & 37 & 57 & 41 \\
\hline & NU & 0.229594 & 0.202736 & 0.191687 & 0.192322 \\
\hline & GC & 0.522502 & 0.545327 & 0.73535 & 0.682739 \\
\hline & $\mathrm{AV}$ & 0.376048 & 0.374031 & 0.463519 & 0.437531 \\
\hline \multirow{4}{*}{ valley } & $\mathrm{T}$ & 39 & 33 & 40 & 30 \\
\hline & NU & 0.260608 & 0.255654 & 0.51246 & 0.422429 \\
\hline & GC & 0.508132 & 0.512626 & 0.628247 & 0.601973 \\
\hline & $\mathrm{AV}$ & 0.38437 & 0.38864 & 0.570354 & 0.512201 \\
\hline \multirow{4}{*}{$\begin{array}{l}\text { Neighborhood } \\
\text { valley }\end{array}$} & $\mathrm{T}$ & $28 n=7$ & $26 n=3$ & $29 n=3$ & $26 n=3$ \\
\hline & NU & 0.3940330 & 0.36191 & 0.705011 & 0.517064 \\
\hline & GC & 0.445856 & 0.473041 & 0.502545 & 0.563509 \\
\hline & $\mathrm{AV}$ & 0.419945 & 0.417476 & 0.603778 & 0.540287 \\
\hline \multirow{4}{*}{$\begin{array}{c}\text { Variance and } \\
\text { intensity contrast }\end{array}$} & $\mathrm{T}$ & $42 \lambda=0.05$ & $37 \lambda=0$ & $57 \lambda=0.05$ & $41 \lambda=0$ \\
\hline & $\mathrm{NU}$ & 0.229594 & 0.202736 & 0.191687 & 0.192322 \\
\hline & GC & 0.522502 & 0.545327 & 0.73535 & 0.682739 \\
\hline & AV & 0.376048 & 0.374031 & 0.463519 & 0.437531 \\
\hline \multirow{4}{*}{$\begin{array}{l}\text { Variance } \\
\text { discrepancy } \\
\text { technique }\end{array}$} & $\mathrm{T}$ & $30 \alpha=0.9$ & $28 \alpha=1$ & $56 \alpha=0.7$ & $36 \alpha=1$ \\
\hline & $\mathrm{NU}$ & 0.498296 & 0.330033 & 0.205936 & 0.285656 \\
\hline & GC & 0.456252 & 0.486657 & 0.732443 & 0.653163 \\
\hline & AV & 0.412636 & 0.408345 & 0.469189 & 0.469 \\
\hline
\end{tabular}

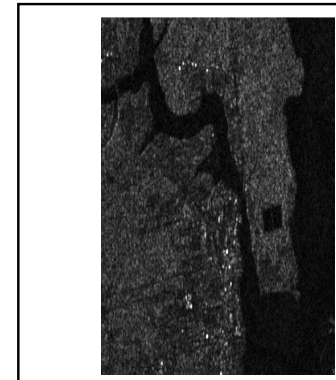

(a)

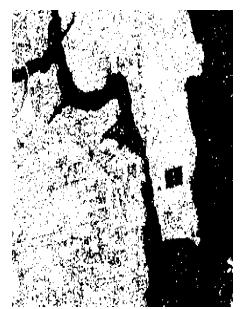

(d)

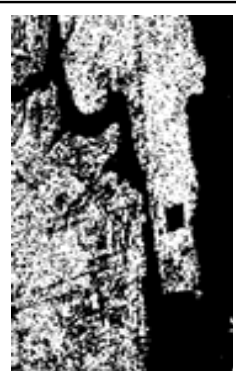

(b)

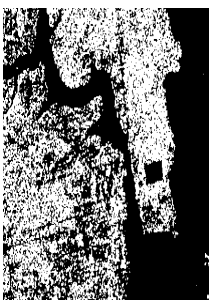

(e)

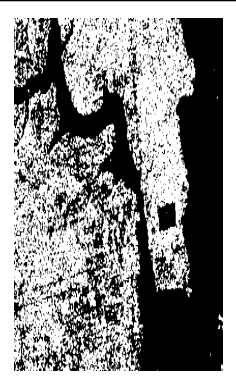

(c)

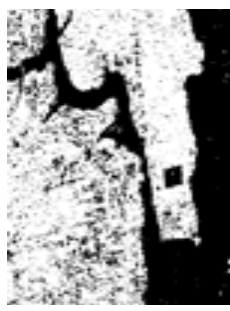

Figure 3. (a) Original image, (b) Otsu technique $\mathrm{T}=42$.(c) Valley emphasis technique $\mathrm{T}=39$. (d) Neighborhood valley emphasis $\mathrm{T}=28$, (e) Variance and intensity contrast $\mathrm{T}=42$, (f) Variance discrepancy $\mathrm{T}=30$. 


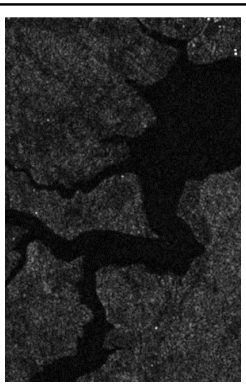

(a)

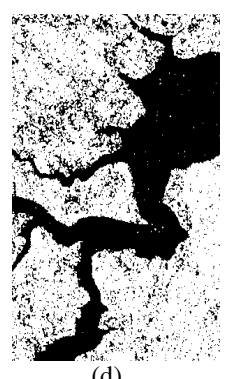

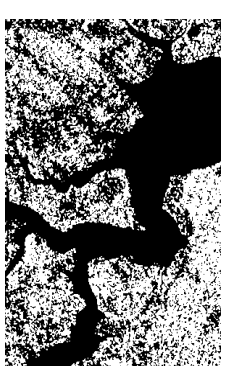

(b)

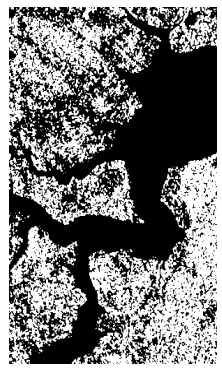

(e)

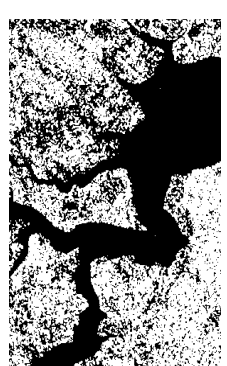

(c)

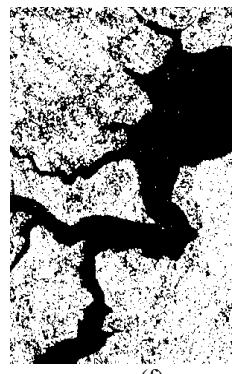

Figure 4.(a) Original image, (b) Otsu technique $\mathrm{T}=37$.(c) Valley emphasis technique $\mathrm{T}=33$.(d) Neighborhood valley emphasis $\mathrm{T}=26$, (e) Variance and intensity contrast $\mathrm{T}=37$, (f) Variance discrepancy $\mathrm{T}=28$.

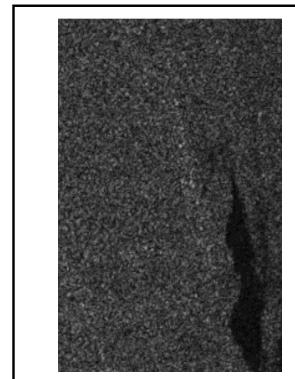

(a)

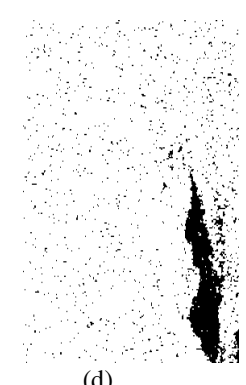

(d)

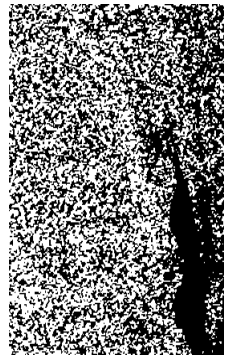

(b)

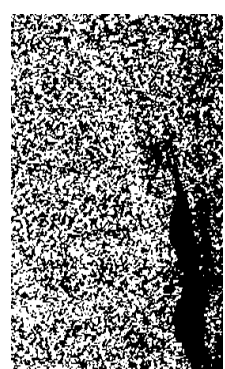

(e)

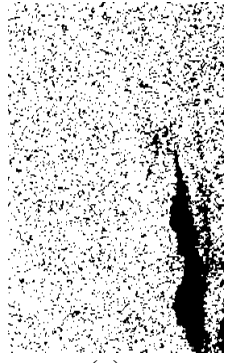

(c)

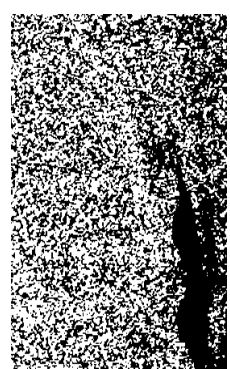

(f)

Figure 5.(a) Original image (b) Otsu technique $\mathrm{T}=57$ (c) Valley emphasis technique $\mathrm{T}=40$, (d) Neighborhood valley emphasis $\mathrm{T}=29$, (e) Variance and intensity contrast $\mathrm{T}=57$ (f) Variance discrepancy $\mathrm{T}=56$ 


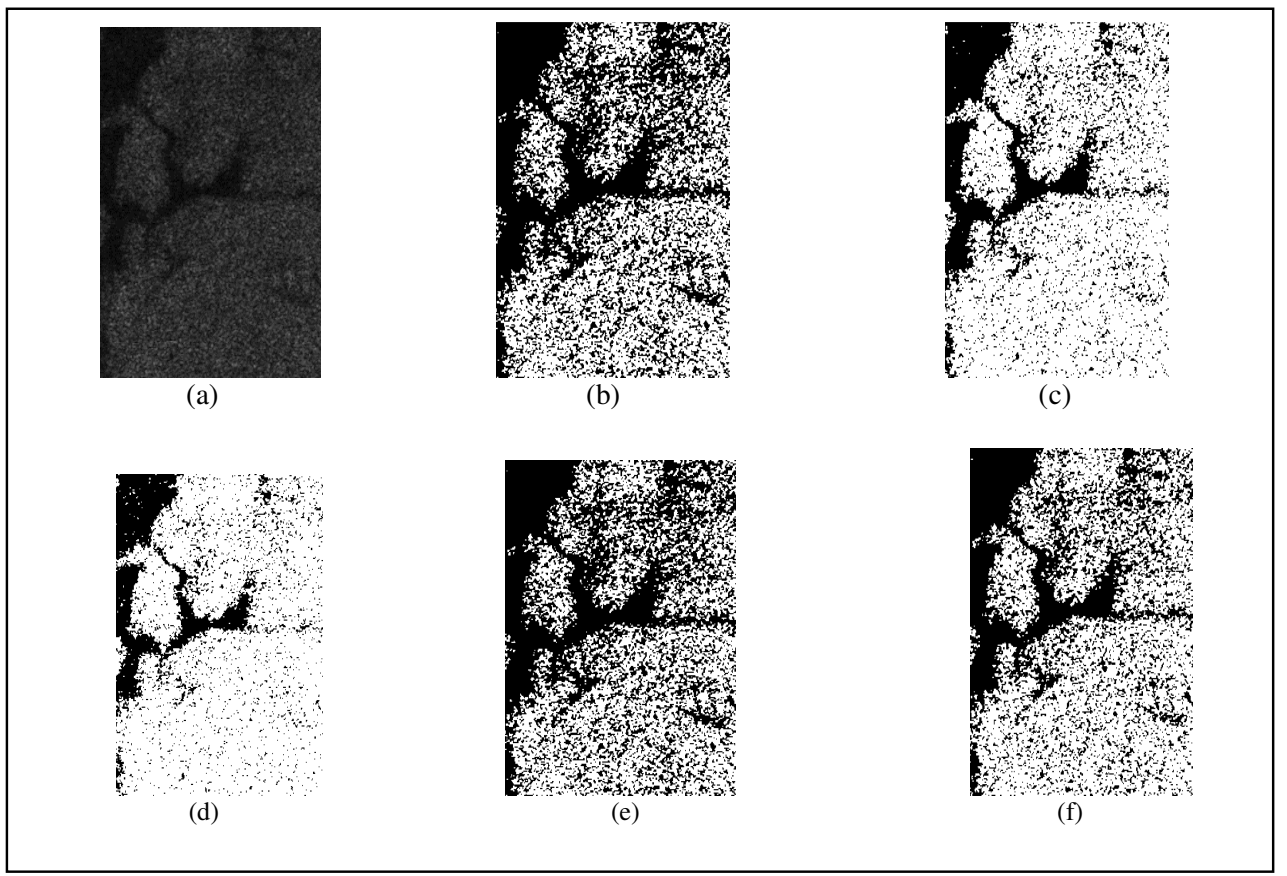

Figure6. (a) original image (b) Otsu technique $\mathrm{T}=41$. (c) Valley emphasis technique $\mathrm{T}=30$ (d) Neighborhood valley emphasis $\mathrm{T}=26$, (e) Variance and intensity contrast $\mathrm{T}=41$.(f) Variance discrepancy $\mathrm{T}=36$.

\section{CONCLUSION AND FUTURE WORK}

Due to the existence of speckle noise SAR images are considered as very accurate and complex images to interpret. Therefore, in this study we present five effective thresholding techniques; these techniques based on between class variance. Furthermore, these thresholding techniques try to solve complex problems for numerous of SAR images. Our contribution in this paper represent with processing SAR images in both high and low contrast. Moreover, our experimental results ensure the efficiency of the five thresholding techniques on thresholded bimodal images, but Otsu method is the best method in presenting the smallest region uniformity (NU), and neighborhood valley emphasis method is the best in presenting inter region contrast (GC).

We aim as a future work for applying the five thresholding techniques on multimodal SAR images; as a try to solve other complex image processing and computer vision problems and applications.

\section{REFERENCES}

[1] D. Samanta, and G. Sanyal." An Approach of Segmentation Technique of SAR Images using Adaptive Thresholding Technique " International Journal of Engineering Research \& Technology (IJERT), Vol. 1 Issue 7, September - 2012.

[2] G.Al-Osaimi and A. El-zaart "Minimum Cross Entropy Thresholding for SAR Images" , IEEE, February 24,2010.

[3] M. Maa, J. Lianga, M. Guoa, Y. Fana, and Y. Yin "SAR image segmentation based on Artificial Bee Colony algorithm" Applied Soft Computing, 112011 ,pp. 5205-5214

[4] Y. Shuai, H. Sun, and G Xu, "A Fast Segmentation Scheme Based on Level Set for SAR Images" IEEE, 2007, pp. 503-506. 
[5] X. Tian, S. Gou and L. Jiao" SAR image segmentation based on spatially adaptive weighted Possibilistic c-means clustering "International Symposium on Intelligent Signal Processing and Communication Systems "Nov.28-Dec.1 2007, pp. 304-307 .

[6] H. Li, and Z. Bai," A New PCNN-Based Method for Segmentation of SAR Images ",10th Intl. Conf. on Control, Automation, Robotics and Vision, Hanoi, 17-20 December 2008, pp. 1635- 1639.

[7] H. B. Kekre , Saylee Gharge and Tanuja K. Sarode " SAR Image Segmentation using Vector Quantization Technique on Entropy Images" (IJCSIS) International Journal of Computer Science and Information Security, Vol. 7, No. 3, March 2010.

[8] N. Otsu, "A threshold Selection Method from Gray Level Histograms", IEEE Transactions on Systems, Man and Cybernetics, vol. SMC-9, no 1, 1979, pp. 62-66.

[9] Hui -Fuang Ng "Automatic thresholding for Defect Detection" Pattern Recognition Letters, 27, 2006 , pp. 1644-1649.

[10] J. Fan, and B. Lei "A modified Valley-Emphasis Method for Automatic Thresholding", Pattern Recognition Letters 33, 2012, pp.703-708.

[11] Y. Qiaoa, Q. Hua, G. Qiana , S. Luob, and W. Nowinskia " Thresholding based on Variance and Intensity Contrast" Pattern Recognition , 40 ,2007,pp. 596 - 608 .

[12]Z. Li, C. Liu, G. Liu ,Y. Cheng , X. Yang ,and C. Zhao” A novel Statistical Image Thresholding Method“, International Journal of Electronics and Communications , vol.64, Dec. 2010, pp.1137-1147.

[13] R. Gonzalez, and R. Woods,” Digital Image Processing”, Prentice-Hall, New Jersey, 2002 , pp1-2 , pp. 567-568.

[14] B. Sankur, and M. Sezgin” Image Thresholding Techniques: A survey over Categories", submitted to Pattern Recognition, 2001 Available on http://busim.ee.boun.edu.tr.

[15] Xian-Bin Wen, Hua Zhang and Fa-Yu Wang, "A Wavelet Neural Network for SAR Image Segmentation", Sensors 2009, 9, pp. 7509-7515.

[16]Lei Hu, Yang Li and Feng Gao ," SAR image segmentation based on Kullback -leibler distance of edge worth " , Advances in Multimedia Information Processing - PCM ,Volume 6297, 2010, pp. 549557.

[17] S. Huang, X. Res, Q. Zhang, and Z. Liu" An improved unsupervised threshold determination method for SAR image change detection", IEEE, vol 2, Oct. 2011,pp. $1704-1707$.

[18] Y. J .Zhang, ”A survey on Evaluation Methods for Image Segmentation”, Pattern Recognition ,Vol.29, no $.8,1996$, pp.1335-133.

\section{Authors}

Ali El-Zaart was a senior software developer at Department of Research and Development, Semiconductor Insight, Ottawa, Canada during 2000-2001. From 2001 to 2004, he was an assistant professor at the Department of Biomedical Technology, College of Applied Medical Sciences, King Saud University. From 2004-2010 he was at Computer Science, College of computer and information Sciences, King Saud University. In 2010, he promoted to associate professor at the same department. Currently, he is an associate professor at the department of Mathematics and Computer Science, Faculty of Sciences; Beirut Arab University. He has published

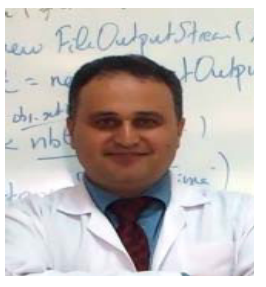
numerous articles and proceedings in the areas of image processing, remote sensing, and computer vision. He received a B.Sc. in computer science from the Lebanese University; Beirut, Lebanon in 1990, M.Sc. degree in computer science from the University of Sherbrook, Sherbrook, Canada in 1996, and Ph.D. degree in computer science from the University of Sherbrook, Sherbrook, Canada in 2001. His research interests include image processing, pattern recognition, remote sensing, and computer vision.

Moumena Al-Bayati Currently is a master student in Beirut Arab University, Department of Mathematics and Computer Science, Beirut, Lebanon (BAU). She received her B.Sc. in computer science 2004 from University of Mosul, Mosul, Iraq.

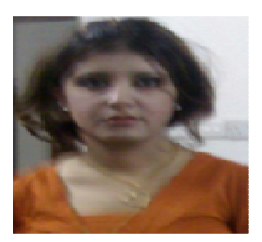

